\section{Recherches sur les Aciers au Cuivre}

Notes présentées à l'Académie des Sciences par M. Pierre Breurl, chef de la section des Métaux au laboratoire du Conservatorre des Arts et Métrers.

\section{I}

Il n'a pas été fait ( $\left.{ }^{*}\right)$, à notre connaissance, d'études méthodiques sur les aciers au cuivre, en vue de leur application à l'industrie. Mushet, Ball et Wigham, Campbell, Arnold, Stead, n'ont abordé que certains points spéciaux de ces études. Nous nous sommes attaché à les entreprendre sur les conseils de M.Vanderheym, et avecle généreux concours des Forges et Aciéries de Firminy. Nos essais ont consisté à faire fabriquer et à étudier des lingots à carburation progressive, contenant des teneur's en cuivre également progressives. Tous ces alliages sont actuellement fabriqués et, pour la plupart, étudiés. Nous donnons ci-après les résultats que nous avons obtenu avec les séries les moins carburées; les autres suivront sous peu.

Les premières séries étudiées, que nous appellerons $\mathrm{A}$ et $B$, avaient la composition survante:

\section{SÉEIE A}

Cunve (pied du lingol). $0,0 \quad 0,490,1,005 \quad 2,015 \quad 3,997 \quad 8,050 \quad 15,97 \quad 31,92$ - (líte du luggot). $0,0 \quad(\ldots$ non dosé . . .) 3,960 7,950 18,91 32 Carbone . ......... $0,1680,1580,1560,1560,1650,1030,1730,150$ Manganèse........ Ĺn ancun cas le manganèze n'a cté supérieur à 0,230 ; il osillait génératement entre 0,10 et 0,15

Shcsum.......... Teneur maxima 0,316 Teneur minima 0,220 Phosphore........ - $\ldots$ - 0,029 - $\ldots$ - 0,020 Sinfre......... - $-0,018$ - $-0,015$

\section{SERIF $B$}

$\begin{array}{lccccccc}1 & 2 & 3 & 4 & 5 & 6 & 7 & 8 \\ 0 & 0,505 & 1,005 & 9 & 025 & 4,009 & 7,960 & 11,015\end{array}$

Cunve (pred du lingon. $0,0 \quad 0,5051,0052,0254,0097,96011,015$ Von — (téte du lingol). 0,0 (. . . non dosé ...) 3,985 7,910 15,985 ci-après Lituone .......... $0,336 \quad 0,390 \quad 0,400 \quad 0,3890,368 \quad 0,372 \quad 0,412 \quad 0,282$ Mangauèse ........ En ancun cas le manganèse n'a été supérieur à 0,230 ; 1 osclllait généraloment entre 0,10 et 0,15

Silcum ......... Teneur maxima 0,316 Teneur minima 0,220

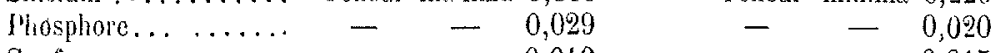
Soutre....... - $\quad-0,010-0,015$

Les analyses ont été faites en prenant des échantillons au pied et à la tète des lingots et on peut reconnaître par leurs résultats que, pour l'acier doux (série A), le cuivre est uniformément réparti dans chaque lingot; il en est de même pour la série $B$ mi-dure, sauf en ce qui concerne le lingot à 32 pour roo de cuivre. Dans ce dernier, on constatait une liquation très marquée; le lingot se divisant à peu près en deux parties, l'une, située près de la tête, contenait 34,2 pour Ioo de cuivre au centre et 2 I, 2 à la surface; l'autre contenait 74,0 pour I oo de cuivre au centre et 24,4 à la surface, et était située vers le pied d'un lingot qu'elle avait avait $p u$ atteindre parce que plus riche en cuivre et, par suite, plus lourde que l'autre.

Les cassures des lingots des deux séries ne contenant pas plus de 4 pour ıo de cuivre étaient sans aucune coloration; à partir de 8 pour 100 de cuivre, elles montraient une coloration rouge d'autant plus intense que la teneur en cuivre était plus grande. Les cassures de ces derniers lingots avaient, près de leur surface, une structure bacillaire que les autres ne possédaient pas.

(1) Séance du 18 juin rgotj.
Tous les lingots ne contenant pas plus de 4 pour ioo de cuivre ont pu être laminés; les autres n'ont pu subir ce travail de façonnage, ils étaient rouverins. Ces faits concordent avec les constatations de Stead et d'autres auteurs. La dureté de ces lingots, déterminée par la méthode de. Brinell, croît avec la teneur en cuivre; les chiffres suivants. sont des moyennes.

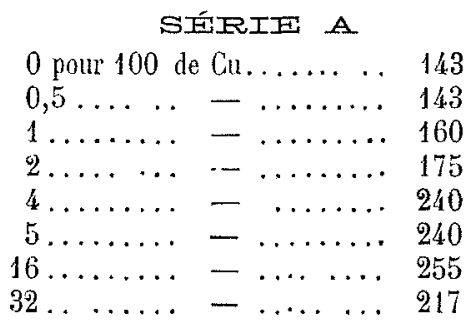

SERTE B

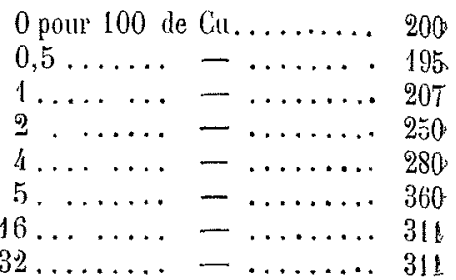

Le cuivre élève donc considérablement la dureté de l'acier: les résultats des autres essais le comfirmeront.

Points singuliers. - Sauf les essais de M. Osmond, nous ne connaissons pas d'autres expériences relatives aux points singuliers de l'acier au cuivre. Nous avons étudié, à ce point de vue, tous les précédents lingots au moyen du couple thermo-électrique Le Chatelier, et avec un galvanomètre enregistreur Callendar. Les courbes dérivées des courbes de refroidissement, d'après la méthode de $M$. Osmond, sont remplies de zigzags qui ne sont pas dus au fonctionnement de nos appareils, comme des essais répétés. l'ont prouvé. Si l'on tient compte seulement des points les plus accusés, on aboutıt aux constatations suivantes.

\section{SÉE工正 A}

$00 / 0$ Gu. AR2, vers $780^{\circ} ;$ AR3, 8700, AR4, $1005^{\circ}$

$1 \ldots-A R 2,715^{\circ} ; A R 3,850^{\circ} ; A R 4,980^{\circ}$.

2.. - ART, $655^{\circ} ;$ AR2, 7300;AR3, 8200;AR4, $1000^{\circ}$.

4.. - ARL et AR2?,6700; AR3, 7800.

$8 \ldots$ - AR0, 5100;AR1, AR2, AR3??; 7300 (point long); ARk, 980o.

16... - Polnt très long à 7400. Les aulres nous parassent incomprchensibles. Point à 10400 correspondant à la fusion du curvre hbre ou d'us alluge cuiveux

$32 \ldots$ - Toint très long a $675^{\circ}$. Les autres nous paraissent incomprébensbles. Pont a $10 \% 0^{\circ}$ correspondaut a la fusion du cuire libre ou d'un allage curreux.

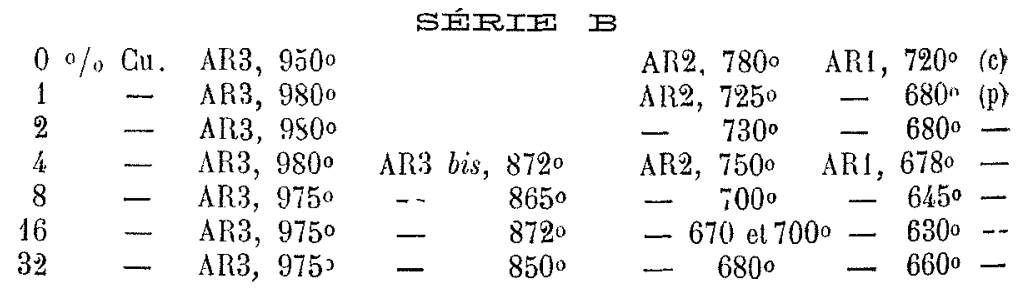

(c) court; (p) plus long que le précédent. Pour les trois derniers, point vers $1040^{\circ}$ correspondant à la fusion du cuivre libre ou d'un alliage de cuivre et de fer très fortement cuivreux.

La série A ne nous permet pas de tirer des conclusions fermes; cependant le cuivre nous paraît faire grossir le point AR I $_{\text {et }}$ baisser AR3, conformément aux constatations de M. Osmond; pour la série B, les essais montrent nettement que le point AR I baisse beaucoup quand la teneur en cuivre augmente et ce point grossit; le point trouvé à $97^{5-980^{\circ}}$ pour tous les aciers nous paraît correspondre à la séparation de la cémentite comme Arnold le suppose pour les aciers ordinaires; AR2 baisse avec l'augmentation du cuivre. Tous ces alliages sont magnétiques à froid, même la partie liquatée fortement cuivreuse de l'alliage de la série $\mathrm{B}$ à 32 pour roo de cuivre (qui l'est peu d'ailleurs). 


\section{II}

Dans une précédente communication, nous avons fait connaître les points singuliers d'aciers au cuivre titrant o, i 5 à 0,18 et 0,35 à 0,38 de carbone; voici les points singuliers d'aciers titrant 0,56 à 0,79 de carbone $\left(^{*}\right)$.

Depuis 0,5 jusqu'à 20 pour 100 de cuivre le point AR I varie peu; il se tient entre $575^{\circ}$ et $600^{\circ}$; un acier au carbone de cette carburation a, généralement, son point $A R_{1}$ vers $670^{\circ}$ à $680^{\circ}$. Le cuivre tait donc très notablement baisser ce point sans toutefois avoir, pour cet abaissement, une action aussi considérable que le nickel ou le manganèse.

On constate le phénomène de récalescence, c'est-à-dire l'abaissement, puis le relèvement de la température à ce point, AR I, d'autant mieux que la teneur en cuivre de l'acier est plus grande; dès que le cuivre s'isole dans l'ácier on constate un point vers $1000^{\circ}$. Cette séparation du cuivre ou d'un alliage fer-cuivre a lieu déjà pour une teneur de 3 pour roo de cuivre, mais cependant la finesse des globules ainsi isolés n'a pas empêché l'élaboration des lingots avant 5 et ro pour rou de cuivre: le métal n'est rouverin qu'audessus de ro pour ivo de cuivre.

Essais de traction des aciers au cuivre. - Les résultats suivants ont été obtenus avec des barrettes normales prises dans les aciers laminés, puis traitées de différentes façons (voir le grand tableau ci-contre).

Ces essais prouvent qu'on aurait grand tort de se fier uniquement aux essais de traction sur barrettes brutes de laminage pour porter une appréciation sur les divers aciers. Tous ces essais montrent bien que le cuivre augmente la ténacité et diminue la ductilité de l'acier, mais dans des proportions éminemment variables avec le traitement du métal.

\section{III}

Essais au choc sur barreaux entaillés $\left(^{* *}\right)$. - Ces essais ont été faits avec l'entaille au tour, préconisée par M. Vanderheym. Le diamètre à fond d'entaille était de ro $\mathrm{mm}$ et l'entaille était aiguë; le mouton pesait $10 \mathrm{~kg}$ et rompait toujours d'un seul coup l'éprouvette.

\begin{tabular}{|c|c|c|c|c|c|}
\hline $\begin{array}{l}\text { Nature } \\
\text { des aciers }\end{array}$ & $\begin{array}{c}\text { Cuivre } \\
\text { en pour } 100\end{array}$ & $\begin{array}{c}\text { Métaux } \\
\text { bruts } \\
\text { de laminage } \\
\text { en } \mathrm{kgm}: \mathrm{mm}^{2}\end{array}$ & $\begin{array}{l}\text { Mélaux } \\
\text { recuts } \\
\text { à } 900^{\circ}\end{array}$ & $\begin{array}{c}\text { Métaux } \\
\text { lrempés } \\
\text { a } 830^{0}-870^{\circ}\end{array}$ & $\begin{array}{l}\text { Métaux } \\
\text { trempés } \\
\text { et revelus } \\
\text { a }: 00^{\circ}\end{array}$ \\
\hline $\begin{array}{c}\text { Série à } \\
0,15-0,18 \\
\text { de } G\end{array}$ & $\begin{array}{l}0,0 \\
0,5 \\
1,0 \\
2,0 \\
4,0\end{array}$ & $\begin{array}{r}16,0 \\
16,0 \\
7,0 \\
7,0 \\
10,0\end{array}$ & $\begin{array}{l}6,6 \\
6,3 \\
6,3 \\
4,8 \\
8,6\end{array}$ & $\begin{array}{r}22,8 \\
20,3 \\
7,9 \\
9,9 \\
11,4\end{array}$ & $\begin{array}{l}22,8 \\
21,6 \\
17,6 \\
10,6 \\
11,4\end{array}$ \\
\hline $\begin{array}{c}\text { Série à } \\
0,35-0,38 \\
\text { de G }\end{array}$ & $\begin{array}{l}0,0 \\
0,5 \\
1,0 \\
2,0 \\
4,0\end{array}$ & $\begin{array}{r}6,0 \\
12,0 \\
7,0 \\
6,0 \\
6,0\end{array}$ & $\begin{array}{l}3,6 \\
0,6 \\
1.6 \\
0,6 \\
0,6\end{array}$ & $\begin{array}{l}4,6 \\
3,1 \\
2,6 \\
0,6 \\
2,6\end{array}$ & $\begin{array}{c}2,6 \\
2,1 \\
1,4 \\
1) \\
2,6\end{array}$ \\
\hline $\begin{array}{c}\text { Série d } \\
0,56-0,79 \\
\text { de C }\end{array}$ & $\left\{\begin{array}{r}0,5 \\
1,0 \\
3,0 \\
10,0\end{array}\right.$ & $\begin{array}{l}n \\
3,1 \\
2,1 \\
)\end{array}$ & $\begin{array}{l}9,6 \\
1,9 \\
2,6 \\
4,1\end{array}$ & $\begin{array}{c}1,1 \\
n \\
1,1\end{array}$ & $\begin{array}{l}3,0 \\
2,1 \\
4,0 \\
3,0\end{array}$ \\
\hline
\end{tabular}

Ces essais au choc, assez concluants pour les aciers à faible carburation, le sont très peu pour les aciers durs qui

\begin{tabular}{|c|c|c|c|c|c|}
\hline & $\begin{array}{c}\text { Curvre } \\
\text { en } \\
\text { centièmes }\end{array}$ & $\begin{array}{c}\text { Limi'e } \\
\text { apparente } \\
\text { d'élastucité } \\
\text { en } \mathrm{kg}: \mathrm{mm}^{2}\end{array}$ & $\begin{array}{c}\text { Clarige } \\
\text { mavim } \\
\text { en } \mathrm{kg} \mathrm{mm}^{2}\end{array}$ & $\begin{array}{c}\text { Allongement } \\
\text { en } \\
\text { centrèmes }\end{array}$ & Striction \\
\hline \multicolumn{6}{|c|}{ I. - Aciers bruts de lamin.tae } \\
\hline $\begin{array}{c}\text { Série à } \\
0,15-0,18 \\
\text { de C }\end{array}$ & $\left\{\begin{array}{l}0(1) \ldots \\
0,5 \ldots \\
1,0 \ldots \\
2,0 \ldots \\
4,0 \ldots\end{array}\right.$ & $\begin{array}{l}44,6 \\
38,8 \\
11,5 \\
47,5 \\
69,5\end{array}$ & $\begin{array}{l}51,1 \\
17,1 \\
49,5 \\
62,6 \\
71,1\end{array}$ & $\begin{array}{l}14,5 \\
25,5 \\
26,5 \\
16,0 \\
13,0\end{array}$ & $\begin{array}{l}0,52 \\
0,66 \\
0,60 \\
0,585 \\
0,465\end{array}$ \\
\hline $\begin{array}{c}\text { Série à } \\
0,35-0,38 \\
\text { de C }\end{array}$ & $\left\{\begin{array}{l}0 . \\
0,5 \ldots \\
1,0 \ldots \\
2,0 \ldots \\
4,0 \ldots\end{array}\right.$ & $\begin{array}{l}37,6 \\
48,6 \\
45,3 \\
68,5 \\
81,8\end{array}$ & $\begin{array}{l}58,1 \\
65,5 \\
64,5 \\
79,5 \\
97,1\end{array}$ & $\begin{array}{l}23,2 \\
20,0 \\
20.5 \\
11,0 \\
11,2\end{array}$ & $\begin{array}{l}0,51 \\
0,485 \\
0,173 \\
0,315 \\
0,235\end{array}$ \\
\hline $\begin{array}{c}\text { Série d } \\
0,56-0,79 \\
\text { de } C\end{array}$ & $\left\{\begin{array}{r}0,5 \ldots \\
1,0 \ldots \\
3,0 \ldots \\
10,0 \ldots\end{array}\right.$ & $\begin{array}{r}62,1 \\
55,3 \\
98,1 \\
102,2\end{array}$ & $\begin{array}{r}83,5 \\
89,7 \\
113,5 \\
125,5\end{array}$ & $\begin{array}{r}12,0 \\
12,5 \\
3,0 \\
2,5\end{array}$ & $\begin{array}{l}0,22 \\
0,169 \\
0,125 \\
0,013\end{array}$ \\
\hline \multicolumn{6}{|c|}{ II. - ACIERS ReCCITS a $900^{\circ}$ ENVIRON } \\
\hline $\begin{array}{l}\text { Série à } \\
0,15-0,18 \\
\text { de } \mathrm{C}\end{array}$ & $\left\{\begin{array}{l}0, \ldots \ldots \\
0,5 \ldots \\
1,0 \ldots \\
2,0 \ldots \\
4,0 \ldots\end{array}\right.$ & $\begin{array}{l}25,2 \\
26,9 \\
38,6 \\
41,3 \\
46,0\end{array}$ & $\begin{array}{l}38,7 \\
41,0 \\
49,0 \\
49,5 \\
50,3\end{array}$ & $\begin{array}{l}30,3 \\
28,0 \\
26,0 \\
25,0 \\
22,0\end{array}$ & $\begin{array}{l}0,03 \\
0,60 \\
0,57 \\
0,58 \\
0,63\end{array}$ \\
\hline $\begin{array}{c}\text { Sérıe a } \\
0,35-0,38 \\
\text { de C }\end{array}$ & $\left\{\begin{array}{l}0 \ldots \ldots \\
0,5 \ldots \\
1,0 \ldots \\
2,0 \ldots \\
4,0 \ldots\end{array}\right.$ & $\begin{array}{l}31,8 \\
33,4 \\
40,9 \\
45,0 \\
56,5\end{array}$ & $\begin{array}{l}51,1 \\
51,7 \\
60,6 \\
61,0 \\
68,5\end{array}$ & $\begin{array}{l}24,0 \\
23,5 \\
20,0 \\
18,0 \\
16,0\end{array}$ & $\begin{array}{l}0,48 \\
0,43 \\
0,10 \\
0.40 \\
0,49\end{array}$ \\
\hline $\begin{array}{c}\text { Série à } \\
0,56-0,79 \\
\text { de C }\end{array}$ & $\left\{\begin{array}{r}0,5 . \\
1,0 . \\
3,0 \ldots \\
10,0 . .\end{array}\right.$ & $\begin{array}{l}11,0 \\
42,3 \\
54,0 \\
65,2\end{array}$ & $\begin{array}{l}71,2 \\
75.5 \\
81.2 \\
82,5\end{array}$ & $\begin{array}{r}16,5 \\
8,5 \\
7,5 \\
10,0\end{array}$ & $\begin{array}{l}0,427 \\
0,182 \\
0,206 \\
0,300\end{array}$ \\
\hline
\end{tabular}

III. - Aciers trempés a $870^{\circ}$ et $830^{\circ}$ Non revenus

\begin{tabular}{|c|c|c|c|c|c|}
\hline \multirow{5}{*}{$\begin{array}{l}\text { Série à } \\
0,15-0,18 \\
\text { de } \mathrm{C}\end{array}$} & 0. & 49,7 & $68, \overline{1}$ & 14,6 & 0,67 \\
\hline & 0,5 . & 49,5 & 67,0 & 16,0 & 0,527 \\
\hline & $1,0$. & 83,0 & 92,8 & 5,55 & 0,26 \\
\hline & & 106,0 & 112,0 & 5,20 & \\
\hline & 4,0 & 106,0 & 138,0 & 7 , & 0,295 \\
\hline \multirow{4}{*}{$\begin{array}{c}\text { Série d } \\
0,35-0,38 \\
\text { de } \mathrm{C}\end{array}$} & 0. & 81,5 & $101, \bar{z}$ & 3.5 & \\
\hline & 0,5 & $76, \overline{3}$ & $76, \overline{5}$ & 1,4 & a moins \\
\hline & $\begin{array}{l}1,0 . \\
9\end{array}$ & 102.5 & 102,5 & 1,4 & oilés \\
\hline & 4.8 & 100,2 & 100,2 & 1,4 & a \\
\hline
\end{tabular}

Séile à $0,5 \ldots$

$0,56-0,79\left\{\begin{array}{l}1,0 \ldots \\ 3,0 \ldots \\ 0,0 \ldots\end{array}\right.$ Plus ou moins volés on tapés à la trempe.

de $\mathrm{C}(10,0 \ldots)$

IV. - Aciers trempés a $870^{\circ}$ ou $830^{\circ}$ Revevus vers $300^{\circ}$

\begin{tabular}{|c|c|c|c|c|c|}
\hline $\begin{array}{c}\text { Série à } \\
0,15-0,18 \\
\text { de } C\end{array}$ & $\begin{array}{l}0 \ldots \ldots \\
0.5 \ldots \\
1,0 \ldots \\
2,0 \ldots \\
4,0 \ldots\end{array}$ & $\begin{array}{r}46,2 \\
51,2 \\
58,8 \\
102,5 \\
85,5\end{array}$ & $\begin{array}{r}64,0 \\
72,2 \\
69,0 \\
111,0 \\
100,0\end{array}$ & $\begin{array}{r}10,5 \\
8,5 \\
11,5 \\
6,5 \\
11,0\end{array}$ & $\begin{array}{l}0,68 \\
0,60 \\
0,68 \\
0,338 \\
0,52\end{array}$ \\
\hline $\begin{array}{c}\text { Série à } \\
0,35 \cdot 0,38 \\
\text { de C }\end{array}$ & $\begin{array}{l}0 \ldots \ldots \\
0, \ldots \ldots \\
1,0 \ldots \\
2,0 \ldots \\
4,0 \ldots\end{array}$ & $\begin{array}{r}72,8 \\
1352 \\
149,0 \\
155,2 \\
153,0\end{array}$ & $\begin{array}{r}99,5 \\
139,0 \\
162,2 \\
167,0 \\
173,0\end{array}$ & $\begin{array}{l}7,5 \\
3,5 \\
3,5 \\
2,0 \\
1,5\end{array}$ & $\begin{array}{l}0,332 \\
0,18 \\
0,75 \\
0,012 \\
0,01\end{array}$ \\
\hline S & & 158,0 & 458,0 & 0,0 & 0,0 \\
\hline
\end{tabular}

$0,56-0,79\left\{\begin{array}{l}1,0 \ldots \\ 3,0 \ldots\end{array}\right.$

de $\mathrm{C}\left\{\begin{array}{r}3,0 \ldots \ldots \\ 10,0 \ldots\end{array}\right\}$ Plus ou moins tapés à la trempe.

(1) Barre écrouie par laminage. 
puraissent tous également fragiles; les essais avec barreaux non entaillés sont beaucoup plus explicites; mais leurs détails nous entraîneraient trop loin; nous nous contenterons de dire que le cuivre ne donne que très peu de fragilité aux aciers étudiés.

Essais de torsion. - Ces essais ont été faits avec une machine permettant de déterminer les moments à 1/10 de $\mathrm{kgm}$ pıès; les barreaux avaient $18 \mathrm{~mm}$ de diamètre et I $00 \mathrm{~mm}$ de longueur cylındrıque. Les résultats sont les suivants; ils portent sur les métaux bruts de laminage:

\begin{tabular}{|c|c|c|c|c|}
\hline Nature des aciers & $\begin{array}{l}\text { Cuivre } \\
\text { en pour } 100\end{array}$ & $\begin{array}{l}\text { Limite } \\
\text { d'élastucité } \\
\text { en kgm }\end{array}$ & $\begin{array}{l}\text { Ffforts } \\
\text { de rupture } \\
\text { en kघm }\end{array}$ & $\begin{array}{c}\text { Angle } \\
\text { de torson } \\
\text { a la rinture } \\
\text { poul } \\
\text { wue longueur } \\
\text { de too nmm. } \\
\text { en degrés }\end{array}$ \\
\hline Série douce. . & $\begin{array}{l}0,0 \\
0,5 \\
1,0 \\
2,0 \\
1,0\end{array}$ & $\begin{array}{l}31,0 \\
33,3 \\
312 \\
47,0 \\
66,0\end{array}$ & $\begin{array}{l}58,0 \\
56,0 \\
59,0 \\
67,0 \\
83,0\end{array}$ & $\begin{array}{l}700 \\
770 \\
925 \\
702 \\
185\end{array}$ \\
\hline Série m-dure. & $\begin{array}{l}0,0 \\
0,5 \\
10 \\
2,0 \\
4,0\end{array}$ & $\begin{array}{l}39,0 \\
35,1 \\
43,2 \\
52,5 \\
69,5\end{array}$ & $\begin{array}{l}67,5 \\
61,5 \\
68,5 \\
79,0 \\
98,0\end{array}$ & $\begin{array}{l}605 \\
196 \\
416 \\
296 \\
183\end{array}$ \\
\hline Série dure... & $\begin{array}{r}0,5 \\
1,0 \\
3,0 \\
10,0\end{array}$ & $\begin{array}{c}58,5 \\
63,0 \\
64,0 \\
30 \text { paulleuse }\end{array}$ & $\begin{array}{r}90,0 \\
104,0 \\
108,0 \\
30,0\end{array}$ & $\begin{array}{r}272 \\
218 \\
116 \\
8\end{array}$ \\
\hline
\end{tabular}

Les essais de torsion amènent aux mêmes conclusions que ceux de traction : le cuivre augmente beaucoup la limite d'élasticité et le moment de rupture dès qu'une teneur de 2 pour 100 est dépassée; la déformation, quoique amoindrie par la présence du cuivre, reste bonne.

Essais dedureté. - Ces essais ont été faits par la méthode de Brinell; la bille avait Io mm de diamètre, la charge employée était de $3000 \mathrm{~kg}$. Les résulltats obtenus sont les suivants:

\begin{tabular}{|c|c|c|c|c|c|}
\hline \multirow[b]{2}{*}{ Séries } & \multirow[b]{2}{*}{$\begin{array}{c}\text { Cuivre } \\
\text { en } \\
\text { pour }: 100\end{array}$} & \multicolumn{4}{|c|}{ Aclers } \\
\hline & & $\begin{array}{c}\text { bruts } \\
\text { de } \\
\text { lammage }\end{array}$ & $\begin{array}{l}\text { reculs } \\
\text { atu roure } \\
\text { cerise }\end{array}$ & $\mid \begin{array}{c}\text { trempés } \\
\text { à } 830^{\circ}-870 \circ \\
\text { non } \\
\text { revenus }\end{array}$ & $\begin{array}{l}\text { trempés } \\
\text { a } 830 \text { a-870 } \\
\text { puis revenus } \\
\text { a } 3000-350\end{array}$ \\
\hline Sénie douce & $\begin{array}{l}0,0 \\
0,5 \\
1,0 \\
2,0 \\
4,0\end{array}$ & $\begin{array}{l}143 \\
146^{\circ} \\
146 \\
202 \\
255\end{array}$ & $\begin{array}{l}124 \\
143 \\
146 \\
174 \\
183\end{array}$ & $\begin{array}{l}207 \\
511 \\
311 \\
311 \\
351\end{array}$ & \\
\hline $\begin{array}{c}\text { Série } \\
\text { mi-dure }\end{array}$ & $\begin{array}{l}0,0 \\
5,5 \\
1,0 \\
2,0 \\
4,0\end{array}$ & $\begin{array}{l}166 \\
209 \\
207 \\
269 \\
302\end{array}$ & $\begin{array}{l}166 \\
166 \\
196 \\
207 \\
212\end{array}$ & $\begin{array}{l}460 \\
627 \\
600 \\
817 \\
782\end{array}$ & $\begin{array}{l}418 \\
460 \\
495 \\
495 \\
782\end{array}$ \\
\hline Série dure & $\begin{array}{r}0,5 \\
1,0 \\
3,0 \\
10,0\end{array}$ & $\begin{array}{l}255 \\
302 \\
418 \\
430\end{array}$ & $\begin{array}{l}228 \\
223 \\
223 \\
241\end{array}$ & 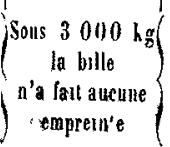 & $\begin{array}{l}555 \\
555 \\
600 \\
782\end{array}$ \\
\hline
\end{tabular}

Les duretés suivent assez bien les résistances à la traction; elles sont généralement supérieures à celles des aciers au nickel de même carburation et ayant subi le même traitement thermique.

Essats de corrosion. - Ces essais n'ont été cffectués que sur les aciers bruts de moulage. Le liquide corrosif était consritué par une dissolution, à volumes égaux, d'cau distillée et d'acide sulfurique à $66^{\circ}$ Beaumé ; les échantillons étaient disposés de telle façon que les sels provenant de l'attaque du métal par le liquide ne vinssent pas recouvrir ce métal; les essais ont duré un mois; on a déterminé les pertes de poids des échantillons; ces pertes sont les suivantes:

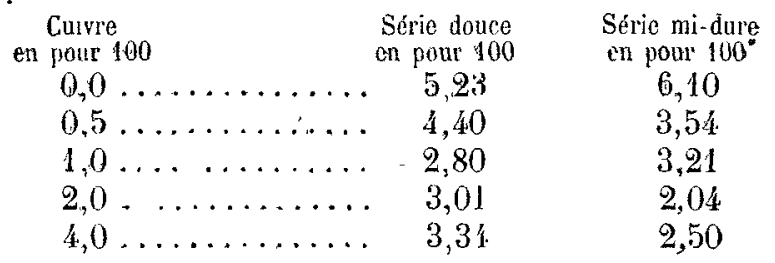

Les pertes des aciers au cuivre sont donc considérablement plus faibles que celles des mêmes aciers sans cuivre; c'est là une importante constatation, analogue à celle faite à propos des aciers au nickel justement réputés à ce point de vue.

Essais au rouverin. - Aucun des aciers ayant de 8 à 4 pour r oo de cuivre n'est rouverin.

Micrographie des aciers au cuivre. - Nos conclusions sur cette question confirment celles de $M$. Stead. A partir de 4 pour roo de cuivre environ, il s'isole dans les lingots des nodules rouges à forte teneur en cuivre, mais le phénomène est d'autant plus marqué que l'acier est plus carburé; ce qui caractérise surtout les aciers au cuirre utilisables (c'est-à-dire jusqu'à 4 pour r oo de cuivre) c'est la finesse de leur structure; ces aciers ont d'autant plus de perlite granulo-sorbitique que la teneur en cuivre est plus élevée; cette sorbite donne de l'homogénéité, de la ténacité et de la dureté au noétal; elle paraît en faire un métal d'une carburation plus haute. Le cuivre se dissolvant dans la ferrite laisse encore à celle-ci beaucoup de malléabilité et, par suite: de cette dissolution, le carbome, trouvant la possibilité de former un carbure plus divisé et en plus grande quantité, apporte, de ce fait, une action durcissante; en conséquence, l'ensemble est à la fois aussi dur que l'acier d'une carburation supérieure, mais est beaucoup moins cassant que lui.

Au point de vue de la nature des constituants, ces aciers. n'ont rien de particulier par rapport aux aciers ordinaires, mais la forme, la distribution, la quantité de ces constituants sont telles que les propriétés spéciales ci-dessus indiquées les signalent à l'artention des métallurgistes; an même titre que les aciers contenant du nickel, ou d'autres élémentsqui sont généralenent d'un prix plus élevé que le cuivre.

\section{Conservation et Dégradation de l'Énergie}

Il y a quatre-vingts ans que Carnot, dans ses "Réflexions sur la puissance motrice du feu et sur les machines propres à développer cette puissance ", a énoncé son célèbre théorème, et sur les débris de la théorie inexacte qui est à la base de ses travaux, l'on a construit une loi générale de la nature. Sorti d'un travall presque technique, le second principe de la thermodynamique s'étend à presque toutes les transformations physiques, et seulement pour les phénomènes où intervient l'énergie rayonnante, l'on a des doutes sur la légitimité de son. application. 